\title{
Cardiac and Respiratory Motion Correction for Simultaneous Cardiac PET/MR
}

\author{
Christoph Kolbitsch ${ }^{1,2}$, Mark A. Ahlman ${ }^{3}$, Cynthia Davies-Venn ${ }^{3}$, Robert Evers ${ }^{3}$, Michael Hansen ${ }^{4}$, Devis Peressutti ${ }^{1}$, \\ Paul Marsden ${ }^{1}$, Peter Kellman ${ }^{4}$, David A. Bluemke ${ }^{3}$, and Tobias Schaeffter ${ }^{1,2}$ \\ ${ }^{1}$ King's College London, Division of Imaging Sciences and Biomedical Engineering, London, United Kingdom; ${ }^{2}$ Physikalisch- \\ Technische Bundesanstalt (PTB), Berlin, Germany; ${ }^{3}$ National Institutes of Health, Clinical Center, Radiology and Imaging Sciences, \\ Bethesda, Maryland; and ${ }^{4}$ National Institutes of Health, National Heart, Lung, and Blood Institute, Bethesda, Maryland
}

\begin{abstract}
Cardiac PET is a versatile imaging technique providing important diagnostic information about ischemic heart diseases. Respiratory and cardiac motion of the heart can strongly impair image quality and therefore diagnostic accuracy of cardiac PET scans. The aim of this study was to investigate a new cardiac PET/MR approach providing respiratory and cardiac motion-compensated MR and PET images in less than 5 min. Methods: Free-breathing 3-dimensional MR data were acquired and retrospectively binned into multiple respiratory and cardiac motion states. Three-dimensional cardiac and respiratory motion fields were obtained with a nonrigid registration algorithm and used in motion-compensated MR and PET reconstructions to improve image quality. The improvement in image quality and diagnostic accuracy of the technique was assessed in simultaneous ${ }^{18}$ F-FDG PET/MR scans of a canine model of myocardial infarct and was demonstrated in a human subject. Results: MR motion fields were successfully used to compensate for in vivo cardiac motion, leading to improvements in full width at half maximum of the canine myocardium of $13 \% \pm 5 \%$, similar to cardiac gating but with a $90 \% \pm 57 \%$ higher contrast-to-noise ratio between myocardium and blood. Motion correction led to an improvement in MR image quality in all subjects, with an increase in sharpness of the canine coronary arteries of $85 \% \pm 72 \%$. A functional assessment showed good agreement with standard MR cine scans with a difference in ejection fraction of $-2 \% \pm 3 \%$. MR-based respiratory and cardiac motion information was used to improve the PET image quality of a human in vivo scan. Conclusion: The MR technique presented here provides both diagnostic and motion information that can be used to improve MR and PET image quality. Reliable respiratory and cardiac motion correction could make cardiac PET results more reproducible.
\end{abstract}

Key Words: simultaneous PET/MR; motion compensation; cardiac and respiratory motion; cardiac $\mathrm{PET}$; radial phase encoding

J Nucl Med 2017; 58:846-852

DOI: 10.2967/jnumed.115.171728

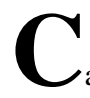

ardiac PET is a highly versatile medical imaging technique $(1,2)$, allowing for the assessment of cardiac viability or perfusion

\footnotetext{
Received Jan. 12, 2016; revision accepted Nov. 17, 2016.

For correspondence contact: Christoph Kolbitsch, Physikalisch-Technische Bundesanstalt (PTB), Medical Physics and Metrological Information Technologies, Abbestrasse 2-12, 10587 Berlin, Germany.

E-mail: christoph.kolbitsch@ptb.de

Published online Feb. 9, 2017.

COPYRIGHT (C 2017 by the Society of Nuclear Medicine and Molecular Imaging.
}

using different tracers. Recently, studies have also shown that plaque in coronary arteries can be detected by NaF PET scans (3). The main challenge for cardiac PET in clinical practice is the compensation of physiologic motion, such as respiratory and cardiac movements of the heart. A range of methods has been developed to overcome this problem. A robust and straightforward approach is motion gating, where data are only used for the final image reconstruction if they have been acquired in a certain motion state such as end-expiration for respiratory gating or middiastolic rest period for cardiac gating $(4,5)$. The disadvantage of this approach is that only a small percentage of the acquired data are used for image reconstruction, leading to a low signal-to-noise ratio. This is often compensated for by acquiring more data, leading to longer scan times.

To use all the acquired data, motion-correction approaches have been proposed. For this, the list-mode data are subdivided into different motion states (i.e., binned), and a motion model is used to transform these to a single reference state before the data are combined. Motion correction is achieved either by combining transformed images after image reconstruction (6), as a preprocessing step before image reconstruction $(7,8)$ or during an iterative reconstruction scheme (i.e., motion-corrected image reconstruction [MCIR]) (9). Several techniques derive motion information directly from PET data $(7,8,10-12)$. Nevertheless, this requires sufficient tracer uptake in the region of interest for all subdivided motion states. Anatomic imaging, such as CT data, has been used to overcome this limitation for respiratory motion but it has to be obtained either before or after the PET scan, which does not provide simultaneous motion information (13).

The introduction of simultaneous PET/MR scanners offers the possibility of using simultaneously acquired high-resolution MR information with excellent soft-tissue contrast for motion correction of PET data $(14,15)$.

Several studies have shown that respiratory motion information obtained with MR can strongly improve the quality of PET images $(16,17)$. Furthermore, tagged MR images have been used for cardiac motion correction in phantom experiments (18). However, 3-dimensional (3D) MR tagging is a time-consuming technique, which has not yet found broad clinical use. Previously presented MR techniques providing motion information for PET/MR yield either respiratory or cardiac motion information and usually no diagnostic images $(19,20)$.

The aim of this work was to investigate a new practical MR technique to obtain anatomic images together with respiratory and cardiac motion information in less than $5 \mathrm{~min}$, which can be used for MCIR of MR images with diagnostic utility and can also be 
immediately applied to PET MCIR. The proposed method was evaluated in vivo in a canine infarct model and demonstrated in in vivo human ${ }^{18} \mathrm{~F}$-FDG PET/MR scans. The effect of MCIR on high-resolution MR and PET image quality was assessed-that is, localization of tracer uptake in the myocardium and contrastto-noise ratio (CNR) between myocardium and blood.

\section{MATERIALS AND METHODS}

MR and PET data were acquired simultaneously during freebreathing and without electrocardiogram synchronization in canine and human subjects. In a first step, the MR data were binned on the basis of a respiratory self-navigator, and respiratory motion was estimated (21). A second step included respiratory motion compensation, and the MR data were binned on the basis of the electrocardiogram to determine nonrigid cardiac motion. In a final step, combined respiratory and cardiac motion information was used in MCIR and provided high-resolution 3D MR anatomic information and complementary 3D functional PET images. A visual summary of this approach is shown in Figure 1.

\section{Infarct Model}

The proposed technique was evaluated in a previously established canine infarct model (22). Five canine subjects (age, 10-13 mo) were imaged $58 \pm 4 \mathrm{~d}$ after they underwent a 90-min surgical occlusion of the left anterior descending coronary artery followed by reperfusion, which caused a subendocardial infarct and subsequent myocardial fibrosis. The main aim of the canine study was to perform multimodality noninvasive imaging of myocardial fibrosis and to assess the sensitivity of several modalities for detecting chronic myocardial scar.
The study was approved by the National Institutes of Health Animal Care and Use Committee and Department of Veterinary Resources.

\section{MR Data Acquisition}

Golden radial phase encoding (GRPE) is a Cartesian sampling scheme. The phase encoding (PE) positions in the 2-dimensional (2D) $\mathrm{PE}_{\mathrm{y}}-\mathrm{PE}_{\mathrm{z}}$ plane were obtained in a radial pattern rather than on a Cartesian grid (23). The angle between 2 successively acquired GRPE lines is the Golden angle of $111.24^{\circ}$. This led to a homogeneous coverage of the 2D PE plane over time and allowed for retrospective combination of data based on respiratory or electrocardiogram information. Because of the radial distribution of the PE lines, images with high quality could be reconstructed even from very few lines using iterative non-Cartesian reconstruction methods. More details on the data acquisition scheme are provided in Supplemental Figure 1 (supplemental materials are available at http://jnm.snmjournals.org).

\section{MR In Vivo Experiments}

The GRPE trajectory was implemented on a 3-T Biograph mMR scanner (Siemens Healthcare). T1-weighted gradient echo images were acquired $5 \mathrm{~min}$ after intravenous injection of $10 \mathrm{~mL}$ of gadofosveset using the following imaging parameters: repetition/echo time, 4.7/2.3 ms; flip angle, $12^{\circ}$; field of view, $288 \mathrm{~mm}^{3}$; voxel size, $1.5 \mathrm{~mm}^{3}$; and total scan time, $4.19 \mathrm{~min}$. Between 3 and 5 channels of a phased array coil were automatically selected for signal reception depending on the positioning of the canine subject. For respiratory motion estimation, data were split into 8 respiratory and 12 cardiac motion states using a soft-gating approach (24).

Before GRPE, standard Cartesian multislice 2D cine scans were acquired covering the entire left ventricle using retrospective cardiac gating during free-breathing. Respiratory motion artifacts were minimized by averaging over 2 consecutive acquisitions. To assess the area of fibrosis, delayed enhancement multislice 2D images were obtained between 12 and 25 min after administration of $10 \mathrm{~mL}$ of gadoteric acid.

All images obtained with the 3D GRPE sampling scheme were reconstructed offline using Matlab (The MathWorks, Inc.) with a nonCartesian iterative sensitivity encoding reconstruction scheme (25). The required coil sensitivity maps were calculated from the data. A temporal and spatial total variation constraint was included into the reconstruction of motionresolved data to take advantage of data redundancy and improve final image quality (26).

\section{Motion Surrogates}

A respiratory motion surrogate was calculated from 1-dimensional inferior-superior projections at $\mathrm{PE}_{\mathrm{y}}=\mathrm{PE}_{\mathrm{z}}=0$ and used for selfnavigation with a temporal resolution of $0.98 \mathrm{~s}$ (21). Because of the limited temporal resolution of the 1-dimensional inferior-superior projections, an external electrocardiogram was used as a cardiac motion surrogate.

\section{Motion Gating and Binning}

On the basis of these motion surrogate signals, the acquired data were separated into several different motion states. Gated images were obtained by restricting data used for the image reconstruction to a single motion state of each motion cycle, which minimizes motion artifacts. 

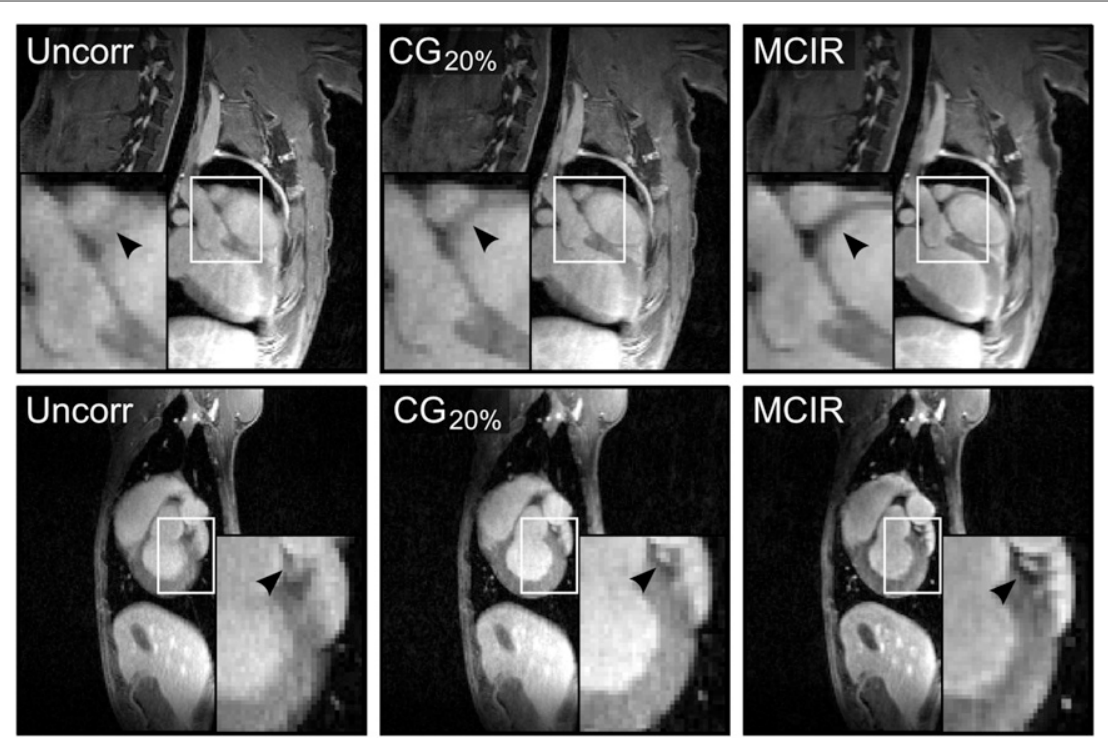

FIGURE 2. Results of MR MCIR. Comparison of uncorrected (Uncorr), cardiac gated $\left(\mathrm{CG}_{20 \%}\right)$, and motion-corrected (MCIR) MR images in 2 different subjects. MCIR leads to a better depiction of the myocardial wall and coronary arteries (black arrows).

For cardiac gating, the windows were positioned in middiastole covering $20 \%\left(\mathrm{CG}_{20 \%}\right)$ or $60 \%\left(\mathrm{CG}_{60 \%}\right)$ of the cardiac cycle independent of the respiratory motion state. In addition, dual-gating (5) was performed, restricting the data used for image reconstruction to $20 \%$ $\left(\mathrm{DG}_{20 \%}\right)$ or $60 \%\left(\mathrm{DG}_{60 \%}\right)$ of a respiratory gating window in endexpiration and a cardiac gating window in middiastole leading to an image reconstruction using $4 \%$ and $36 \%$ of the total data, respectively. In addition, dual gating with a $60 \%$ respiratory and $20 \%$ cardiac gating window was applied $\left(\mathrm{DG}_{60 \%-20 \%}\right)$.

Respiratory binning was performed on the basis of the amplitude of the respiratory surrogate such that each of the 8 motion bins contained the same amount of data. For cardiac binning the cardiac cycle was separated into 12 equally spaced time intervals.

\section{MR Motion Estimation and Motion Correction}

A temporal sparse free form deformation technique was used to estimate nonrigid respiratory and cardiac motion (27). This technique yielded displacement fields for each pixel, describing the forward and backward transformation from a reference image to all other images. Nonrigid MCIR was performed directly during the iterative image reconstruction of the final MCIR MR images by applying motion vector fields at each iteration (28). The reference motion states for all image registrations were end-expiration and middiastole.

In addition, affine respiratory motion parameters were obtained for a region of interest covering the heart using a normalized crosscorrelation-based registration algorithm (29). The affine respiratory motion information was used only to speed up the reconstruction of the cardiac motion-resolved images. All other MCIR reconstructions were performed using nonrigid cardiac and nonrigid respiratory motion information, unless stated otherwise. Affine motion correction can be performed as a preprocessing step before the iterative image reconstruction, requiring less computational time than the iterative nonrigid MCIR approach.

\section{Evaluation of MR Image Quality and Motion Accuracy}

Functional parameters such as end-diastolic volume, end-systolic volume, ejection fraction and myocardial mass were measured in the cardiac motion-resolved images after respiratory motion correction.
To assess the accuracy of respiratory and cardiac motion correction, the sharpness of the right coronary artery was measured in reformatted MR images (30) without motion correction with $\mathrm{CG}_{20 \%}$ and with cardiac and respiratory MCIR. The small diameter of the coronary arteries makes them very susceptible to physiologic motion and therefore they provide a sensitive measure for the performance of MCIR.

\section{PET In Vivo Experiments}

Five canine subjects were injected intravenously with $370 \mathrm{MBq}$ of ${ }^{18} \mathrm{~F}-\mathrm{FDG} 113 \pm$ 36 min before cardiac PET list-mode data were acquired for 5 min simultaneously with the GRPE MR scanning.

\section{PET Image Reconstruction and \\ Motion Correction}

PET images were reconstructed with STIR (Software for Tomographic Image Reconstruction) using an iterative $3 \mathrm{D}$ ordered-subsets expectation maximization algorithm with 23 subsets and 2 full iterations and 4-mm isotropic 3D gaussian postfiltering (31). MCIR was performed during reconstruction to transform voxels to the same motion state at each iteration, which was selected to be middiastole and endexpiration, respectively (9). Motion was only compensated for, if the motion amplitude in the heart was larger than the MR resolution to minimize reconstruction times. The position and size of gating and binning windows were the same as for the simultaneously acquired MR data.

The MR-based attenuation-correction values were acquired during freebreathing. This image is an average over multiple cardiac and respiratory cycles and describes mainly the most common cardiac and respiratory motion state (i.e., middiastole and end-expiration) and therefore agrees well with the reference motion states of MCIR. All PET image reconstructions except MCIR were performed with the unmodified attenuation-correction image. For MCIR it was transformed to match the individual motion states using the MR-based motion information.

\section{Evaluation of PET Images}

The myocardial uptake was analyzed using the American Heart Association 17-segment bull's-eye plots. In addition, full width at half maximum (FWHM) of the tracer uptake in the myocardium was measured at a midposterior position and basal posterior position in reformatted short-axis slices as an average over a $10^{\circ}$ segment. These locations showed high cardiac motion amplitudes in all dogs. CNR values were calculated at these positions as the difference between the peak myocardial signal and the mean blood-pool signal relative to the SD of the bloodpool signal. The analysis was performed for uncorrected, $\mathrm{CG}_{20 \%} \mathrm{CG}_{60 \%}$, and MCIR images. One-tailed paired Student $t$ tests assuming that motion compensation improves image quality were used to compare the results, with a $P$ value smaller than 0.01 considered statistically significant.

\section{Human In Vivo PET/MR Scans}

GRPE and PET data were obtained simultaneously in a female subject $(55$ y) $8 \mathrm{~min}$ after intravenous injection of $20 \mathrm{~mL}$ of gadobenate dimeglumine and $121 \mathrm{~min}$ after intravenous injection of $368.15 \mathrm{MBq}$ of ${ }^{18} \mathrm{~F}-\mathrm{FDG}$. This scan was part of a study to assess vasculitis approved by the National Institutes of Health Institutional Review Board (NCT02257866). For cardiac motion-resolved images, data were split into 8 cardiac motion states to ensure sufficient image quality for accurate cardiac motion estimation. The MR-based attenuation 


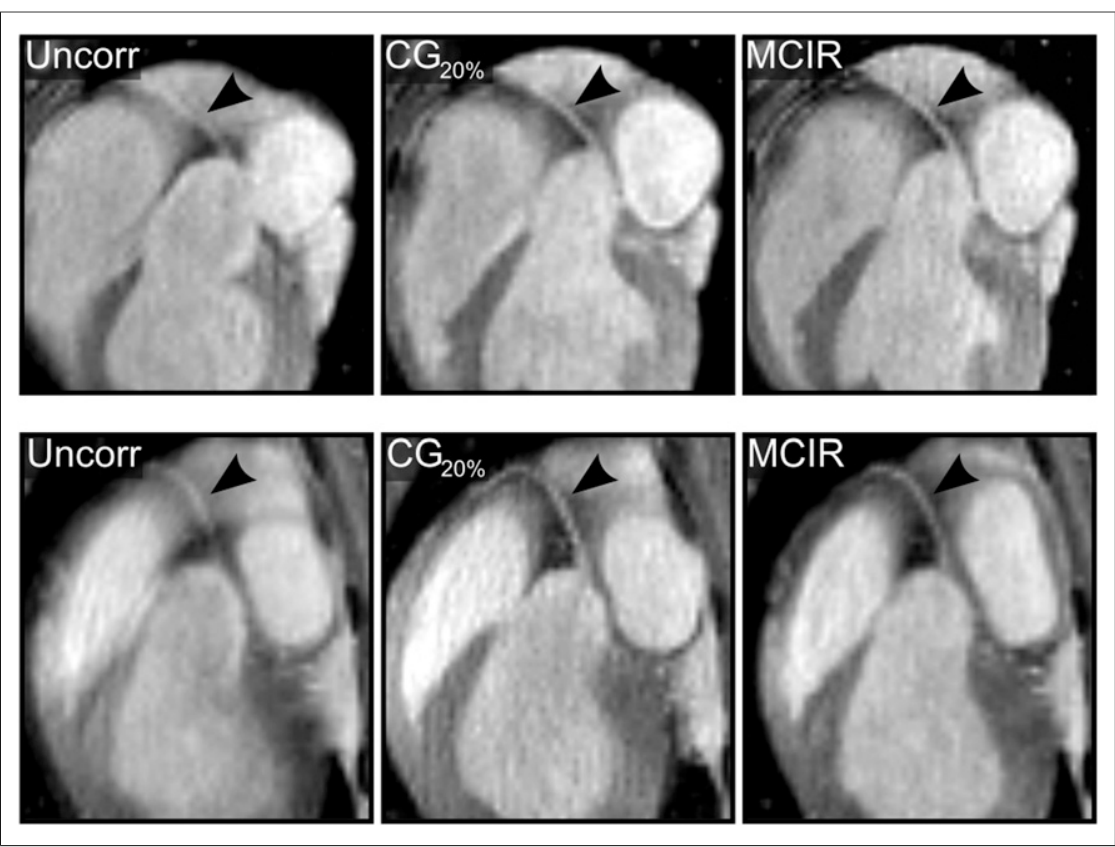

FIGURE 3. Assessment of coronary arteries. Reformatted images show right (black arrow) coronary artery for 2 different subjects for uncorrected (Uncorr), cardiac gated $\left(\mathrm{CG}_{20 \%}\right)$, and motion-corrected (MCIR) MR images.

map was adapted on the basis of the MCIR GRPE image to ensure that the attenuation map and MCIR PET data were in the same motion state. The MCIR MR image was used to semiautomatically redefine the lung-liver and heart-lung boundaries of the attenuation map. All other MR and PET acquisition and reconstruction parameters were the same as for the canine subjects.

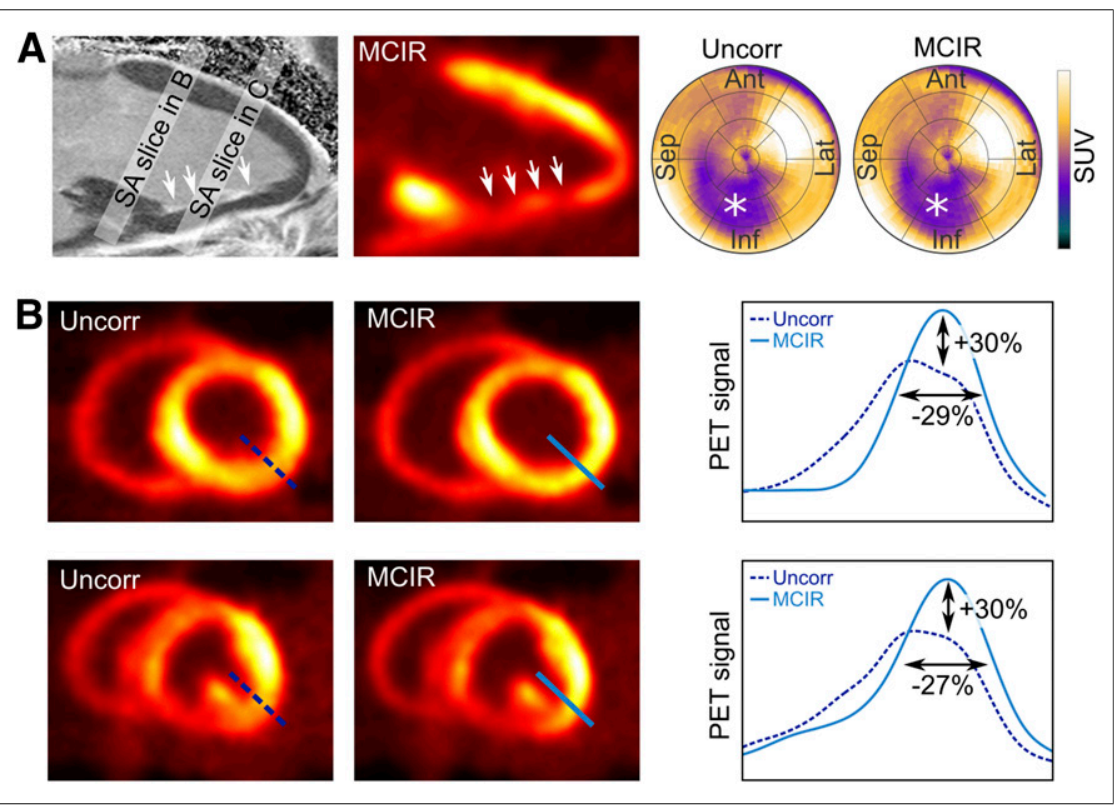

FIGURE 4. Results of in vivo PET scans. (A) Delayed enhancement (DE) 2-chamber MR image showing fibrotic tissue in inferior part of left ventricle (white arrows). This area also shows low myocardial ${ }^{18} \mathrm{~F}-\mathrm{FDG}$ uptake. Bull's-eye plots show low uptake region clearly $\left(^{*}\right)$ in uncorrected (uncorr) and cardiac motion-corrected (MCIR) PET images. (B) Basal and midventricular shortaxis (SA) PET motion-corrupted and MCIR slices. Cardiac motion leads to strong blurring of myocardial uptake in posterior area of ventricle. MCIR can correct for blurring, leading to increase in signal amplitude of $30 \%$ and improvement of FWHM of $29 \%$.

\section{RESULTS}

Results are reported from canine subjects unless explicitly stated otherwise.

\section{MR Assessment of Cardiac Function}

We found good agreement between left ventricular volumes and mass measured on the free-breathing cine dataset compared with the GRPE images binned into 12 cardiac phases, with a difference in end-diastolic volume of $-0.2 \pm 4 \mathrm{~mL}$, in end-systolic volume of $1 \pm 2 \mathrm{~mL}$, in myocardial mass of $2 \pm 8 \mathrm{~g}$, and in ejection fraction of $-2 \% \pm 3 \%$. BlandAltman plots are shown in Supplemental Figure 2.

\section{MR Motion Estimation}

The amplitude of respiratory motion of the heart was small, with an average over all canine subjects of $0.9 \pm 0.3 \mathrm{~mm}$ (maximum, $1.3 \mathrm{~mm})$. For cardiac motion, the motion amplitude was $5 \pm 3 \mathrm{~mm}$, with similar amplitudes perpendicular and parallel to the axis of the left ventricle. On the basis of these findings, only the cardiac motion component was used for MCIR of the PET data. A visualization of cardiac motion fields can be found in Supplemental Figure 3.

\section{MR Motion Correction}

Figure 2 compares uncorrected, $\mathrm{CG}_{20 \%}$, and cardiac and respiratory MCIR MR images. MCIR strongly improved image quality, leading to clearly superior depiction of small features such as the wall of the myocardium and coronary arteries.

Figure 3 shows reformatted images depicting the right and left coronary arteries comparing uncorrected, $\mathrm{CG}_{20 \%}$, and MCIR MR images. An improvement in vessel sharpness of $61 \% \pm 77 \%(P=$ $0.03)$ and $85 \% \pm 72 \%(P=0.006)$ relative to the uncorrected images was achieved with $\mathrm{CG}_{20 \%}$ and MCIR, respectively. MCIR improved the vessel sharpness by $18 \% \pm 12 \%(P=0.012)$ relative to $\mathrm{CG}_{20 \%}$.

\section{PET In Vivo MCIR}

The canine in vivo PET results are depicted in Figure 4. A 2-chamber slice from the delayed enhancement MR scan shows enhancement of the inferior wall of the left ventricle. This region correlates well with low uptake in the corresponding ${ }^{18}$ F-FDG PET scan, although PET seems to overestimate the subendocardial scar. In the bull's-eye plot, the low uptake region is clearly depicted for both the uncorrected and the MCIR approach. 
TABLE 1

Results of In Vivo PET Acquisitions Comparing FWHM and CNR Midposterior and Basal-Posterior Position in Left Ventricle

\begin{tabular}{|c|c|c|c|c|c|c|c|c|c|}
\hline \multirow[b]{2}{*}{ Subject no. } & \multirow[b]{2}{*}{ Position } & \multicolumn{4}{|c|}{ FWHM (mm) } & \multicolumn{4}{|c|}{ CNR } \\
\hline & & Uncorrected & $\mathrm{CG}_{60 \%}$ & $\mathrm{CG}_{20 \%}$ & MCIR & Uncorrected & $\mathrm{CG}_{60 \%}$ & $\mathrm{CG}_{20 \%}$ & MCIR \\
\hline \multirow[t]{2}{*}{1} & Basal posterior & 10.53 & 10.95 & 9.13 & 9.57 & 25.82 & 21.69 & 20.65 & 27.76 \\
\hline & Midposterior & 12.89 & 12.56 & 11.72 & 11.63 & 26.33 & 22.43 & 10.41 & 22.06 \\
\hline \multirow[t]{2}{*}{2} & Basal posterior & 14.57 & 12.33 & 10.35 & 11.13 & 13.43 & 21.12 & 32.20 & 46.74 \\
\hline & Midposterior & 13.43 & 12.42 & 11.38 & 11.43 & 30.83 & 36.66 & 38.38 & 53.87 \\
\hline \multirow[t]{2}{*}{3} & Basal posterior & 13.17 & 12.7 & 11.71 & 11.61 & 22.55 & 18.45 & 23.21 & 31.13 \\
\hline & Midposterior & 12.9 & 12.41 & 11.46 & 11.82 & 49.26 & 48.62 & 42.55 & 77.34 \\
\hline \multirow[t]{2}{*}{4} & Basal posterior & 13.67 & 12.52 & 9.67 & 11.02 & 45.55 & 33.96 & 19.14 & 56.51 \\
\hline & Midposterior & 11.7 & 11.17 & 10.36 & 10.21 & 36.14 & 25.15 & 22.09 & 39.26 \\
\hline \multirow[t]{2}{*}{5} & Basal posterior & 15.69 & 15.52 & 14.77 & 13.91 & 110.62 & 92.04 & 59.34 & 150.23 \\
\hline & Midposterior & 13.83 & 13.77 & 13.77 & 12.4 & 211.33 & 133.13 & 71.53 & 163.84 \\
\hline Mean & & 13.54 & 12.82 & 11.69 & 11.68 & 57.19 & 45.33 & 33.95 & 66.87 \\
\hline SD & & 1.13 & 1.21 & 1.64 & 1.03 & 60.55 & 37.87 & 19.32 & 50.24 \\
\hline
\end{tabular}

Values were determined as average over $10^{\circ}$ segment of ventricle.

Cardiac motion leads to signal blurring, which is visible in basal and midventricular short-axis slices (Fig. 4B). The strongest blurring effect occurs in the posterior part of the ventricle. In this area, the proposed MCIR approach leads to an increase of signal amplitude and an improved FWHM of the myocardial uptake of approximately $30 \%$.

The pooled data from all 5 dogs are summarized in Table 1. Compared with the uncorrected images, MCIR and $\mathrm{CG}_{20 \%}$ achieved a similar improvement in FWHM (sharpness) of $13 \% \pm$ $5 \%(P<0.001)$ and $14 \% \pm 9 \%(P<0.001)$, respectively. MCIR resulted in a similar CNR as the uncorrected reconstruction $(P=0.124)$, which was $90 \% \pm 57 \%(P=0.0054)$ higher than for $\mathrm{CG}_{20 \%}$. $\mathrm{CG}_{60 \%}$ did not lead to a statistically significant improve- ment of FWHM compared with the uncorrected case, probably because of residual systolic motion occurring in the gating window. A summary of all comparisons is given in Supplemental Tables 1 and 2 .

\section{Human In Vivo MR and PET MCIR}

Uncorrected and MCIR MR and PET images are shown in Figure 5. Respiratory and cardiac motion fields are shown in Supplemental Figure 4. The maximum motion amplitudes of the heart were $16 \mathrm{~mm}$ for respiratory and $14 \mathrm{~mm}$ for cardiac motion. Therefore, both nonrigid respiratory and cardiac motion information were used for the final MR and PET MCIR reconstruction. MR image quality was strongly improved using MCIR. MCIR led to a better visualization of the ${ }^{18} \mathrm{~F}$-FDG uptake in the myocardium and an increase in CNR of $103 \%$ and an improvement of FWHM of $18 \%$ compared with the uncorrected PET images. $\mathrm{DG}_{20 \%}, \mathrm{DG}_{60 \%-20 \%}$, and $\mathrm{DG}_{60 \%}$ led to a reduced CNR of $-17 \%,-12 \%$, and $-58 \%$ compared with MCIR.

\section{DISCUSSION}

In this in vivo study, we presented a practical MR technique that yields 3D high-resolution anatomic and functional information in one 5-min scan. Derived respiratory and cardiac motion fields allow for MR and PET MCIR, which significantly improves image quality.

The functional assessment of the left ventricle on the cardiac motion-resolved MR images showed a slight overestimation of the end-systolic volume and thus underestimation of the ejection fraction by $-2 \% \pm$ $3 \%$ compared with the multislice 2D cine. This is probably due to the lower 
temporal resolution of the proposed method, which causes blurring of the fast systolic contraction. Nevertheless, the error has a similar magnitude compared with other $3 \mathrm{D}$ cine studies with higher resolution and scan times of $10 \mathrm{~min}$ (32). As mentioned above, respiratory motion amplitudes of the canine hearts were small, therefore acquiring the multislice $2 \mathrm{D}$ cine data during freebreathing and averaging MR raw data over 2 consecutive acquisitions were considered a good gold standard.

The uptake of ${ }^{18}$ F-FDG in the human myocardium was low, probably because the patient was fasting and underwent a PET/ CT scan before the PET/MR, which prolonged the time between tracer application and imaging. Nevertheless, this demonstrated very well that MCIR can recover PET uptake differences (i.e., between myocardium and blood pool), which are otherwise hardly visible due to motion blurring. This could be especially important for small uptake regions such as $\mathrm{NaF}$ PET scans of coronary plaque (3). $\mathrm{DG}_{20 \%}$ led to poor image quality because of the low number of counts, which would need to be compensated for with longer acquisition times. For $\mathrm{DG}_{60 \%}$, the gating windows were too wide and did not minimize motion artifacts sufficiently.

Changes in the subject's respiratory pattern during data acquisition can make it challenging to provide an accurate motion model for the entire scan. Further studies are required to investigate how these changes can be detected and how the motion modeling process needs to be adapted to accommodate for this situation. For this study, the coil sensitivity information was obtained from the uncorrected MR data. Further improvements in image quality might be achieved by recalculating coil sensitivity maps after each MCIR step.

One limitation of this study is the small number of subjects and because of the small respiratory amplitudes of the canine heart, PET respiratory MCIR could not be evaluated in the animal model. However, the feasibility of MCIR simultaneous PET/MR could be demonstrated and quantified.

So far, random and scatter coincidences were not accounted for in the PET reconstruction. This will be part of future developments.

Several methods have been proposed to obtain motion information directly from ${ }^{18}$ F-FDG PET scans $(7,8,11-13)$. Nevertheless, the accuracy of the estimated motion depends, especially for nonrigid cardiac motion, on sufficient PET uptake in the ventricle. The advantage of our method is that it can provide accurate motion information for different PET tracers independent of the uptake characteristics.

The improvement in measured uptake values of the canine myocardium was up to $24 \%$, which is in good agreement with previous human studies reporting relative differences in the activity concentration and in wall thickness measurement between $20 \%$ and $30 \%$ due to cardiac motion (13). Furthermore, our results suggest that MCIR achieves an improvement in FWHM of PET uptake representing an increase in resolution of up to $30 \%$, which is comparable to $\mathrm{CG}_{20 \%}$. In contrast to cardiac gating, MCIR uses all the available PET data and therefore leads to $90 \%$ higher CNR. In this study, $\mathrm{CG}_{60 \%}$ did not lead to a statistically significant improvement in image quality compared with the uncorrected PET images.

\section{CONCLUSION}

We have presented for the first time, to our knowledge, a practical MR technique, which yields diagnostic and motion information of the heart in less than $5 \mathrm{~min}$. The cardiac and respiratory motion information was successfully applied to improve the quality of MR and simultaneously acquired PET data. This approach could strongly increase image quality and diagnostic accuracy for cardiac PET studies, particularly for lesions of small size and activity.

\section{DISCLOSURE}

This work was supported by the SUBLIMA project (funded by the European Union under the seventh framework program, no. 241711) and by the Centre of Excellence in Medical Engineering (funded by the Wellcome Trust and EPSRC, no. WT 088641/Z/09/ Z). This research was also supported in part by the National Institutes of Health intramural research training program. No other potential conflict of interest relevant to this article was reported.

\section{ACKNOWLEDGMENTS}

We thank Dr. Kris Thielemans for his support with the PET reconstruction and Dr. Peter Grayson for collaborative work in this study.

\section{REFERENCES}

1. Bengel FM, Higuchi T, Javadi MS, Lautamäki R. Cardiac positron emission tomography. J Am Coll Cardiol. 2009;54:1-15.

2. Schindler TH, Schelbert HR, Quercioli A, Dilsizian V. Cardiac PET imaging for the detection and monitoring of coronary artery disease and microvascular health. JACC Cardiovasc Imaging. 2010;3:623-640.

3. Joshi NV, Vesey AT, Williams MC, et al. ${ }^{18} \mathrm{~F}$-fluoride positron emission tomography for identification of ruptured and high-risk coronary atherosclerotic plaques: a prospective clinical trial. Lancet. 2014;383:705-713.

4. Chander A, Brenner M, Lautamäki R, Voicu C, Merrill J, Bengel FM. Comparison of measures of left ventricular function from electrocardiographically gated ${ }^{82} \mathrm{Rb}$ PET with contrast-enhanced CT ventriculography: a hybrid PET/CT analysis. J Nucl Med. 2008;49:1643-1650.

5. Teräs M, Kokki T, Durand-Schaefer N, et al. Dual-gated cardiac PET: clinical feasibility study. Eur J Nucl Med Mol Imaging. 2010;37:505-516.

6. Klein GJ, Reutter BW, Huesman RH. Non-rigid summing of gated PET via optical flow. IEEE Trans Nucl Sci. 1997;44:1509-1512.

7. Livieratos L, Stegger L, Bloomfield PM, Schafers K, Bailey DL, Camici PG. Rigid-body transformation of list-mode projection data for respiratory motion correction in cardiac PET. Phys Med Biol. 2005;50:3313-3322.

8. Feng T, Wang J, Fung G, Tsui B. Non-rigid dual respiratory and cardiac motion correction methods after, during, and before image reconstruction for 4D cardiac PET. Phys Med Biol. 2016;61:151-168.

9. Qiao F, Pan T, Clark JW Jr, Mawlawi OR. A motion-incorporated reconstruction method for gated PET studies. Phys Med Biol. 2006;51:3769-3783.

10. Lamare F, Ledesma Carbayo MJ, Cresson T, et al. List-mode-based reconstruction for respiratory motion correction in PET using non-rigid body transformations. Phys Med Biol. 2007;52:5187-5204.

11. Gigengack F, Ruthotto L, Burger M, Wolters CH, Jiang X, Schafers KP. Motion correction in dual gated cardiac PET using mass-preserving image registration. IEEE Trans Med Imaging. 2012;31:698-712.

12. Le Meunier L, Slomka PJ, Dey D, et al. Motion frozen ${ }^{18} \mathrm{~F}-\mathrm{FDG}$ cardiac PET. $J$ Nucl Cardiol. 2011;18:259-266.

13. Lamare F, LeMaitre A, Dawood M, et al. Evaluation of respiratory and cardiac motion correction schemes in dual gated PET/CT cardiac imaging. Med Phys. 2014;41:072504.

14. Fürst S, Grimm R, Hong I, et al. Motion correction strategies for integrated PET/ MR. J Nucl Med. 2015;56:261-269.

15. Judenhofer MS, Wehrl HF, Newport DF, et al. Simultaneous PET-MRI: a new approach for functional and morphological imaging. Nat Med. 2008;14:459-465.

16. Fayad H, Schmidt H, Wurslin C, Visvikis D. Reconstruction incorporated respiratory motion correction in clinical simultaneous PET/MR imaging for oncology applications. J Nucl Med. 2015;56:884-889.

17. Grimm R, Fürst S, Souvatzoglou M, et al. Self-gated MRI motion modeling for respiratory motion compensation in integrated PET/MRI. Med Image Anal. 2015;19:110-120. 
18. Huang C, Petibon Y, Ouyang J, et al. Accelerated acquisition of tagged MRI for cardiac motion correction in simultaneous PET-MR: phantom and patient studies. Med Phys. 2015;42:1087-1097.

19. Catana C. Motion correction options in PET/MRI. Semin Nucl Med. 2015; 45:212-223.

20. Munoz C, Kolbitsch C, Reader AJ, Marsden P, Schaeffter T, Prieto C. MR-based cardiac and respiratory motion-compensation techniques for PET-MR imaging. PET Clin. 2016;11:179-191.

21. Buerger C, Clough RE, King AP, Schaeffter T, Prieto C. Nonrigid motion modeling of the liver from 3-D undersampled self-gated golden-radial phase encoded MRI. IEEE Trans Med Imaging. 2012;31:805-815.

22. Wu KC, Kim RJ, Bluemke DA, et al. Quantification and time course of microvascular obstruction by contrast-enhanced echocardiography and magnetic resonance imaging following acute myocardial infarction and reperfusion. $\mathrm{J} \mathrm{Am}$ Coll Cardiol. 1998;32:1756-1764.

23. Prieto C, Uribe S, Razavi R, Atkinson D, Schaeffter T. 3D undersampled goldenradial phase encoding for DCE-MRA using inherently regularized iterative SENSE. Magn Reson Med. 2010;64:514-526.

24. Johnson KM, Block WF, Reeder SB, Samsonov A. Improved least squares MR image reconstruction using estimates of k-space data consistency. Magn Reson Med. 2012;67:1600-1608.
25. Pruessmann KP, Weiger M, Boernert P, Boesiger P. Advances in sensitivity encoding with arbitrary k-space trajectories. Magn Reson Med. 2001;46:638-651.

26. Cruz G, Atkinson D, Buerger C, Schaeffter T, Prieto C. Accelerated motion corrected three-dimensional abdominal MRI using total variation regularized SENSE reconstruction. Magn Reson Med. 2016;75:1484-1498.

27. Shi W, Jantsch M, Aljabar P, et al. Temporal sparse free-form deformations. Med Image Anal. 2013;17:779-789.

28. Batchelor PG, Atkinson D, Irarrazaval P, Hill DLG, Hajnal J, Larkman D. Matrix description of general motion correction applied to multishot images. Magn Reson Med. 2005;54:1273-1280.

29. Buerger C, Schaeffter T, King AP. Hierarchical adaptive local affine registration for fast and robust respiratory motion estimation. Med Image Anal. 2011;15:551-564

30. Etienne A, Botnar RM, Van Muiswinkel AMC, Boesiger P, Manning WJ, Stuber M. "Soap-Bubble" visualization and quantitative analysis of 3D coronary magnetic resonance angiograms. Magn Reson Med. 2002;48:658-666.

31. Thielemans K, Tsoumpas C, Mustafovic S, et al. STIR: software for tomographic image reconstruction release 2. Phys Med Biol. 2012;57:867-883.

32. Pang J, Sharif B, Fan Z, et al. ECG and navigator-free four-dimensional wholeheart coronary MRA for simultaneous visualization of cardiac anatomy and function. Magn Reson Med. 2014;72:1208-1217.

\section{Erratum}

There is an error in "Predicting Pathologic Response of Esophageal Cancer to Neoadjuvant Chemotherapy: The Implications of Metabolic Nodal Response for Personalized Therapy" by Findlay et al. (J Nucl Med. 2017;58:266-274). The study interval was May 2006 to January 2014, not May 2006 to November 2014 as stated in the Materials and Methods section. The authors regret the error. 